\title{
Ansiedad y Afrontamiento Cadavérico en Alumnos de Anatomía
}

\author{
Anxiety and Cadaveric Coping in Anatomy Students
}

Martín J. Mazzoglio y Nabar' ${ }^{1}$ Rubén D. Algieri² \& Elba B. Tornese ${ }^{3}$

\begin{abstract}
MAZZOGLIO Y NABAR, M. J.; ALGIERI, R. D \& TORNESE, E. B. Ansiedad y afrontamiento cadavérico en alumnos de anatomía. Int. J. Morphol., 37(3):928-937, 2019.

RESUMEN: En estudios preliminares describimos la importancia clínica e impacto pedagógico del estudio con material cadavérico (MC) en alumnos de Anatomía y hallamos un significativo uso de sustancias psicoactivas (SP), principalmente recursantes y/o alumnos con actividades laborales. La forma de afrontamiento cadavérico (AfC) es un factor de distrés asociado con síntomas (asco, vómitos, alteraciones del sueño), con uso de SP e interfiere el proceso educativo. El objetivo fue evaluar la ansiedad en alumnos con afrontamiento cadavérico negativo. Estudio observacional y transversal mediante encuesta a 740 alumnos que incluyó: Escala de Ansiedad ante la muerte -EAM-, Escala de Ansiedad de Hamilton, preguntas sobre SP y se investigaron términos asociados a conceptos ("material cadavérico", "pieza anatómica"). En alumnos con "afrontamiento cadavérico negativo" (AfCN) (EAM<15, miedo alto/medio con MC y reacciones físicas) se aplicó la Escala de Ansiedad de Hamilton. Se aplicaron parámetros estadísticos, significación p<0,05 y requisitos ético-legales. Objetivamos alta y sostenida prevalencia de alumnos con AfCN, que presentaron mayor puntaje de ansiedad y prevalencia de uso de SP (los ansiolíticos con mayor prevalencia que las sustancias excitatorias); se registró correlación entre cantidad de SP y grado de reacciones negativas $(\mathrm{r}=0,86)$. La ansiedad psíquica fue mayor y se asoció con el grado de miedo. Ratificamos alta prevalencia de AfCN que se asoció con mayor nivel de ansiedad y uso de SP, principalmente ansiolíticos. Los síntomas de ansiedad se asociaron con las dimensiones conceptuales registradas y tienen impacto en el proceso de enseñanza-aprendizaje.
\end{abstract}

PALABRAS CLAVE: Ansiedad; Consumo de sustancias; Afrontamiento cadavérico; Anatomía; Estrés académico.

\section{INTRODUCCIÓN}

En estudios preliminares describimos la importancia clínica, con impacto pedagógico en el aprendizaje, del estudio con material cadavérico (MC) en alumnos de la materia Anatomía de la Carrera de Medicina (Mazzoglio y Nabar et al., 2015a; Mazzoglio y Nabar et al., 2016; Mazzoglio y Nabar et al., 2017a,b; Mazzoglio y Nabar et al., 2018). Hallamos que la utilización de MC en el contexto didáctico de dicha materia se asociaba con un significativo uso de sustancias psicoactivas (SP), principalmente entre los alumnos recursantes y/o aquellos alumnos que desarrollaban actividades laborales (Mazzoglio y Nabar et al., 2011; Mazzoglio y Nabar et al., 2015b; Mazzoglio y Nabar et al., 2017a,b; Mazzoglio y Nabar et al., 2018). El uso de SP referido por los alumnos (cantidad de sustancias utilizadas) presentó una correlación con la carga horaria laboral (cantidad de horas de jornada laboral y cantidad de días de trabajo en la semana) y las exigencias de estudio, siendo mayor en los momentos previos al examen parcial.

La forma de "afrontamiento cadavérico", constructo descripto y acuñado por el Prof. Dr. Daniel R. Algieri y el Dr. Martín Mazzoglio y Nabar (Mazzoglio y Nabar et al.; 2015a), es un factor de distrés que se asocia con reacciones emocionales negativas (Charlton et al., 1994; Snelling et al., 2003; Arraéz-Aybar et al., 2004b) que pueden ser sintomáticas (vómitos, alteraciones del sueño, alteraciones autonómicas como desmayos o lipotimias, necesidad de abandonar el aula de disección), subjetivas (asco, repugnan-

\footnotetext{
${ }^{1}$ Médico. Especialista en Psiquiatría. Especialista en Neurología Cognitiva y Neuropsiquiatría. Magister en Neurociencia y Biología del Comportamiento. Magister en Psicofarmacología. Docente Adscripto de Anatomía y de Psiquiatría y Salud Mental, Facultad de Medicina, UBA. Jefe de Sección Psiquiatría, CPFCABA, Servicio Penitenciario Federal, Buenos Aires, Argentina.

${ }^{2}$ Médico. Especialista en Cirugía General. Doctor en Medicina. Prof. Regular Adjunto de Anatomía y Docente Autorizado de Cirugía, Facultad de Medicina, UBA. Jefe de Departamento, Hospital Aeronaútico Central, Buenos Aires, Argentina.

${ }^{3}$ Médica. Doctora en Medicina, UBA. Médica Psiquiatra y Legista. Prof. Adj. (Eq) de Anatomía, Facultad de Medicina, UBA. Docente Autorizada del Departamento de Psiquiatría y Salud Mental, UBA. Jefe de Servicio, Hospital Moyano, GCABA, Buenos Aires, Argentina.

Laboratorio de Pedagogía y Ciencias de la Educación, $3^{\circ}$ Cátedra de Anatomía, Facultad de Medicina, Universidad de Buenos Aires, Argentina.
} 
cia, temor) o la conducta de utilizar sustancias psicoactivas, tanto ansiolíticas como excitatorias, para disminuir el estrés frente al MC y como una reacción restitutiva. Estas reacciones emocionales negativas configuran una forma de afrontamiento cadavérico negativo (AfCN) que interfiere el proceso educativo y puede alterar el aprendizaje significativo de la materia (Charlton et al.; Babinski et al.; 2003; ArraézAybar et al., 2004a) además de impactar negativamente en la adherencia con la materia y carrera; existen reportes que asocian estas primeras reacciones con las conductas y mecanismos defensivos que los alumnos aplicarán cuando llegan al ciclo clínico con pacientes.

El objetivo del presente trabajo fue evaluar la ansiedad en aquellos alumnos con afrontamiento cadavérico negativo.

\section{MATERIAL Y MÉTODO}

Estudio observacional y transversal mediante la implementación de una encuesta anónima, estructurada y normatizada a 740 alumnos (años 2015, 2016 y 2017) de la materia Anatomía que incluyó la Escala de Ansiedad ante la muerte -EAM- (Templer, 1970) modificada, la Escala de Ansiedad de Hamilton (HARS) (Hamilton, 1959; Lobo et al.; 2002) y preguntas sobre uso de sustancia psicoactivas, parámetros socioeconómicos (edad, sexo, lugar de nacimiento, situación en la materia, aspectos laborales, estudios previos, motivaciones en relación con la elección de la carrera) y se investigaron términos asociados a los conceptos "material cadavérico" y "pieza anatómica" con la técnica de listados libres de Bernard y la técnica de montones para investigar concepciones culturales e imaginarios y grupos de dimensiones conceptuales.

La EAM fue validada según una escala de 15 ítems (verdaderos o falsos) y se determinó que las respuestas que alcancen 15 puntos indican un nivel alto de ansiedad ante la muerte, mientras que los valores cercanos a 30 refieren un nivel bajo. Se definió afrontamiento cadavérico negativo (AfCN) (Mazzoglio y Nabar et al., 2015a) en aquellos alumnos: puntaje menor a 15 en la EAM (parámetro estadístico), miedo alto o medio en la sala de disección (componente subjetivo) y reacciones físicas síntomas vegetativos- asociados con el trabajo con MC (componente somático/objetivo).

En aquellos alumnos con AfCN se aplicó la Escala de Ansiedad de Hamilton -HARS- (Lobo et al.). Fue criterio de exclusión: alumnos que ya habían cursado la materia.
Los resultados fueron procesados con pruebas de estadística descriptiva e inferencial (SPSS para dicho análisis y realización de dendogramas), para análisis de los términos Visual Anthropac Freelists versión: 1.0.1.36 y Visual Anthropac Pilesorts versión: 1.0.2.60; se definió la significación $\mathrm{p}<0,05$.

El presente trabajo cumple con los reparos ético-legales y normativos vigentes (consentimiento informado, cumplimiento de requisitos estipulados por las GCP -Good Clinical Practice-, Disposición ANMAT 6677/10 y adhesión a principios éticos con origen en la Declaración de Helsinski).

\section{RESULTADOS}

El 30,54 \% de los alumnos presentó un afrontamiento cadavérico negativo frente al $69,46 \%$ con afrontamiento cadavérico negativo; sus características poblacionales se exhiben en la Tabla I y Figura 1 para una mejor visualización cualitativa de las diferencias.

Al analizar los alumnos con afrontamiento cadavérico negativo, el 19,91\% refirió miedo alto o muy alto ante los TP con MC y en el 23,89\% fue moderado; sin diferencias entre género y con valores significativamente menores en alumnos sin afrontamiento negativo (Fig. 2). El puntaje promedio en la EAM fue 16,89, en masculinos se objetivó mayor impacto y en femeninas el grado de afectación fue mayor (Fig. 3). Este grupo presentó un puntaje promedio de ansiedad en rango moderado/severo medido con la HARS; observamos que el puntaje de ansiedad psíquica fue mayor al de somática. Destacamos, según promedio de intensidad, los síntomas autonómicos, síntomas cognitivos, el estado de ánimo ansioso y los síntomas cardiovasculares como ítems con mayor afectación (Fig. 4).

Las reacciones físicas más frecuentes fueron la sudoración, el asco y la repugnancia, con un considerable porcentaje de alumnos $(15,04 \%)$ que abandonaron la sala de disección en algún TP; manifestaron haber vivenciado evidencias de disregulación autonómica (palpitaciones, desmayo y temblores) con menor prevalencia que el impacto subjetivo (Fig.5). Se determinó que dichas reacciones se asociaron con en la vida diaria como dificultad para dormir, tener imágenes recurrentes del MC y dificultades para comer determinados alimentos (Fig. 6). Al analizar los factores asociados con mayor impacto, hallamos que el ver MC en piezas anatómicas o "desmembrado" fue el más prevalente y que presentó mayor diferencia entre grupos, seguido por ver zonas anatómicas en particular y la visualización de la cara del MC (Fig. 7). Este grupo de alumnos presentó ma- 


\begin{tabular}{|c|c|c|c|}
\hline & & & \\
\hline & & SIN afrontamiento cadavérico negativo & CON afrontamiento cadavérico negativo \\
\hline \multirow[b]{2}{*}{ Sexo } & Femenino & $55,25 \%$ & $60,18 \%$ \\
\hline & Masculino & $44,75 \%$ & $39,82 \%$ \\
\hline \multirow[b]{2}{*}{ Edad (promedio en años) } & Femenino & $21,32 \%$ & $19,96 \%$ \\
\hline & Masculino & $20,61 \%$ & $21,25 \%$ \\
\hline \multirow[b]{2}{*}{ Lugar de vivienda de nacimiento } & Urbana & $78,79 \%$ & $79,20 \%$ \\
\hline & \begin{tabular}{|l|l|l} 
Rural \\
\end{tabular} & $21,21 \%$ & $20,80 \%$ \\
\hline \multirow{3}{*}{ Experiencias previas en familiares cercanos o amigos } & fallecimiento & $20,04 \%$ & $17,26 \%$ \\
\hline & enfermedad severa o terminal & $79,96 \%$ & $82,74 \%$ \\
\hline & Si & $48,25 \%$ & $65,04 \%$ \\
\hline Actividad laboral & No & $51,75 \%$ & $34,96 \%$ \\
\hline \multirow[b]{5}{*}{ Cuántas horas trabaja durante la semana? } & $<12 \mathrm{hs} / \mathrm{semana}$ & $12,06 \%$ & $11,50 \%$ \\
\hline & 12 a 20 horas/semana & $22,76 \%$ & $27,43 \%$ \\
\hline & 20 a 30 horas/semana & $26,07 \%$ & $26,11 \%$ \\
\hline & 30 a 40 horas/semana & $20,62 \%$ & $21,68 \%$ \\
\hline & $>40$ horas/semana & $18.48 \%$ & $13,27 \%$ \\
\hline
\end{tabular}
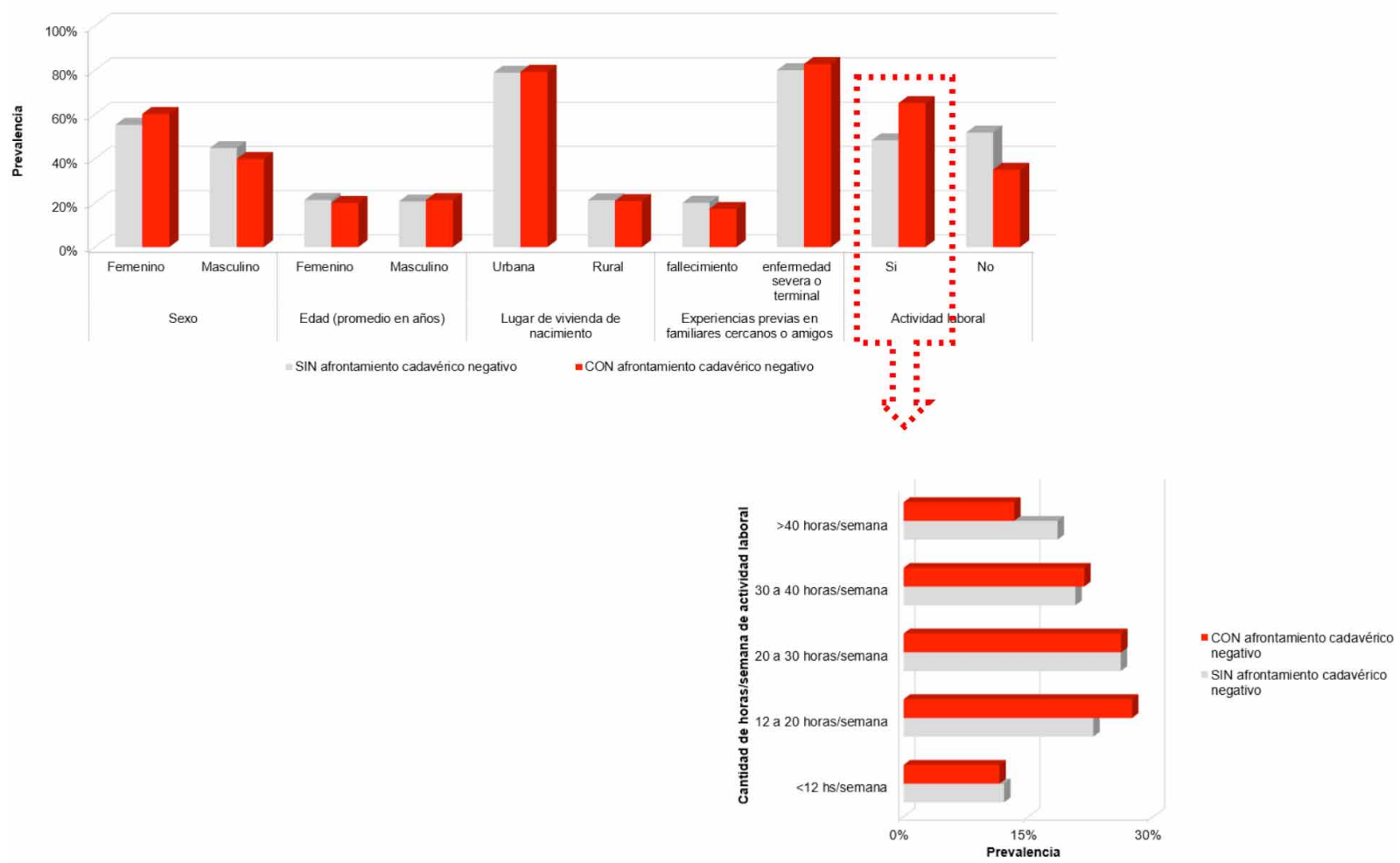

Tabla 1 y Fig. 1. Características poblaciones de la muestra con especificación de los alumnos con afrontamiento cadavérico negativo

yor prevalencia de uso de sustancias para dormir $(29,65$ $\%)$ con mayor incremento de éstas desde el inicio del ciclo lectivo $(15,04 \%)$ y un $7 \%$ menos de horas de sueño; se destacó el uso de benzodiacepinas $(67,16 \%$, el doble de aquellos sin afrontamiento negativo) (Fig. 8). Casi el $40 \%$ de los alumnos con AfCN utilizó alguna sustancia psicoactiva para aumentar sus horas de estudio, su acceso fue por recomendación de un compañero o información de internet y se destacó, según frecuencia, el uso de: bebidas energizantes, café, modafinilo y cafiaspirina ${ }^{\circledR}$ (Fig. 9). Entre quienes presentaron AfCN la prevalencia de uso de sustancias y reacciones físicas fueron mayores $(\mathrm{p}<0,05)$, y hubo correlación entre cantidad de sustancias consumidas y el grado de reacciones negativas $(r=0,85)$.

En el análisis de las dimensiones conceptuales, ha- llamos diferencias en aquellos alumnos con AfCN. Observamos que el término "material cadavérico" lo asociaron más con muerte/repugnancia y asco/sucio; y "pieza anatómica" con desmembramiento y rotura (Fig. 10).

En el grupo de alumnos con AfCN objetivamos que las horas de sueño presentaban correlación inversa con la cantidad de SP utilizadas $(\mathrm{r} 2=0,88)$ y la cantidad de SP fue correlativa con el promedio de puntaje en la subescala de ansiedad psíquica (r2 $=0,77)$ (Fig. 11) y con el grado de reacciones negativas $(r=0,86)$ (Fig. 12). La ansiedad psíquica fue mayor y se asoció con el grado de miedo $(r=0,76)$. La cantidad de horas de sueño referida fue menor en alumnos con AfCN, mientras que el número promedio de sustancias utilizadas para aumentar las horas de estudio fue un $60 \%$ mayor que los alumnos sin AfCN (Fig. 13). 


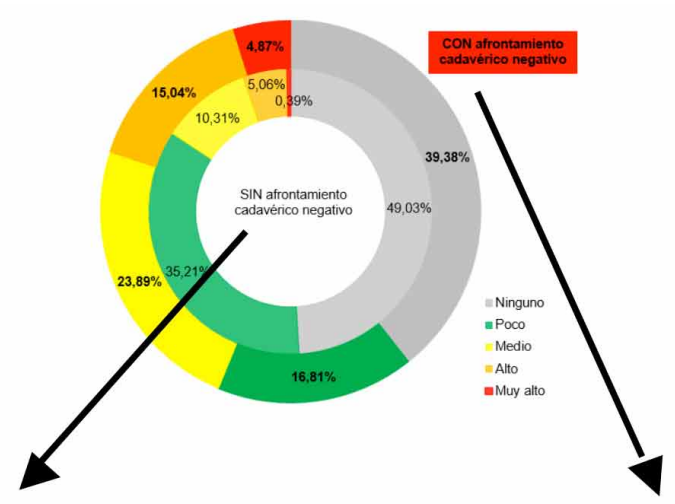

SIN afrontamiento cadavérico negativo

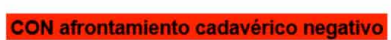

$100 \%$

$0 \%$
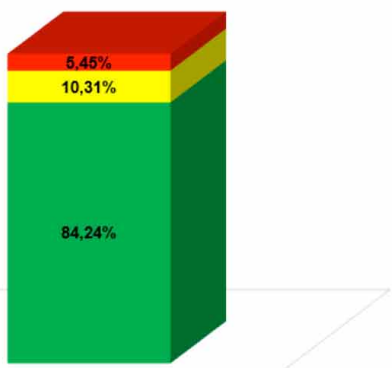

Fig. 2. Grado de miedo en escala de 3 y 5 niveles, con especificación de los grupos SIN y CON afrontamiento cadavérico negativo

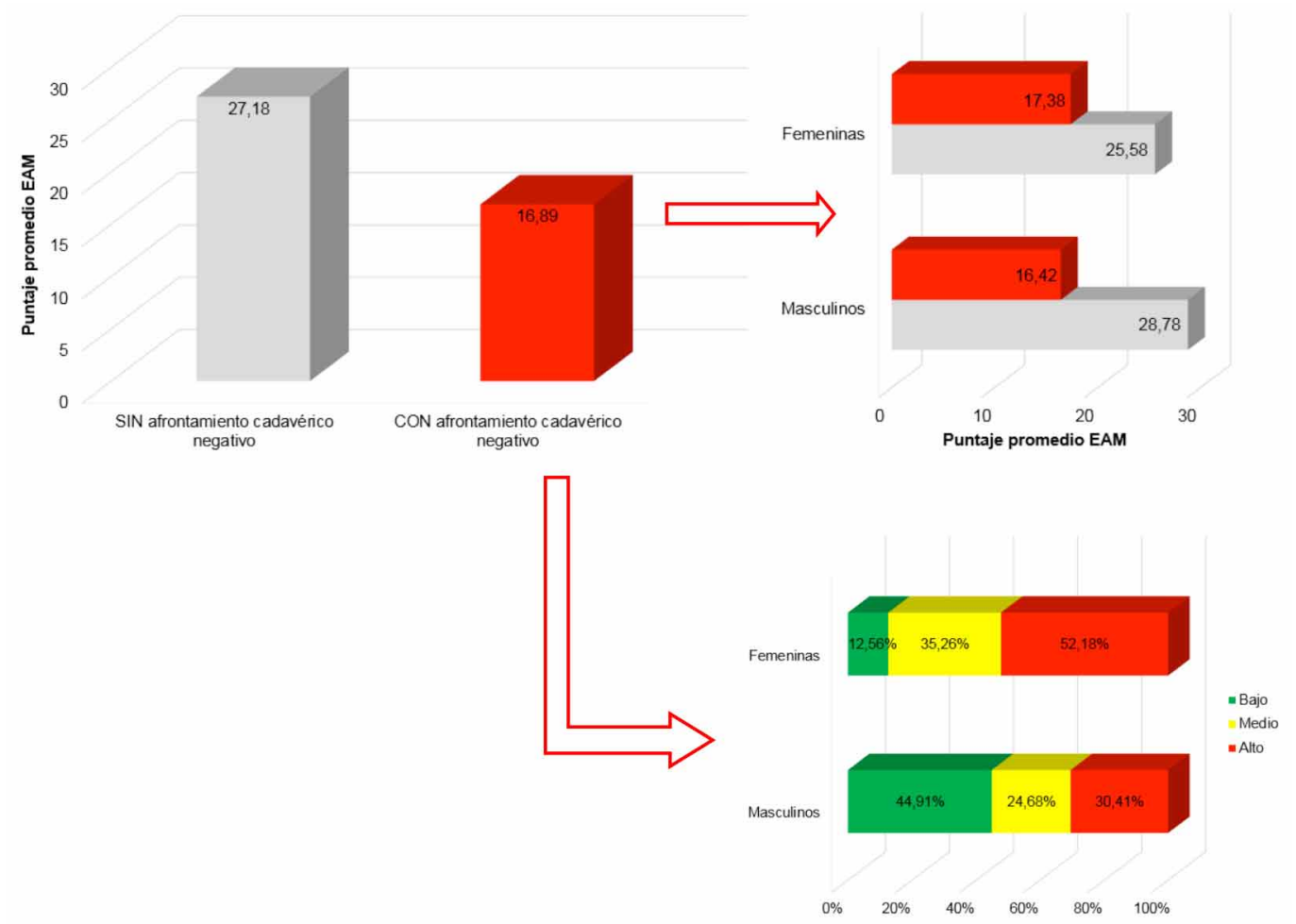

Fig.3. Promedio de puntaje en la EAM (Escala de ansiedad ante la muerte de Templer, 1970) con especificación del género y del grado de miedo. 

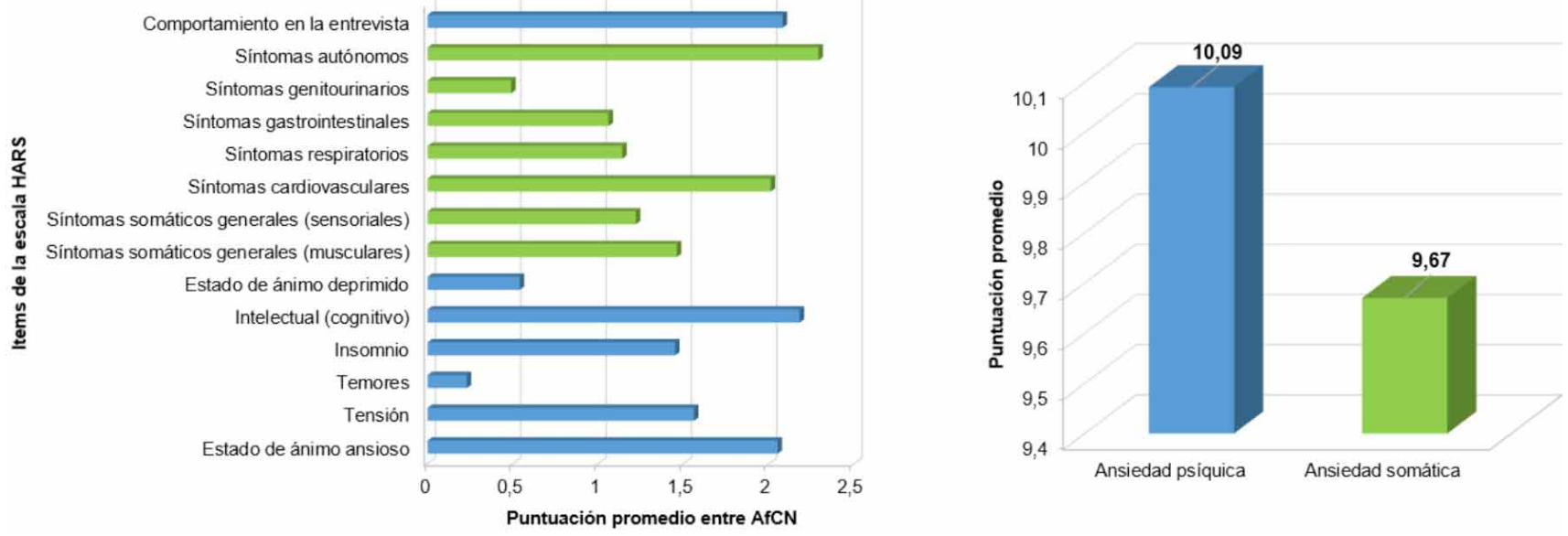

Fig. 4. Promedio de puntuaciones obtenidas en la HARS y resultado de las subescalas.

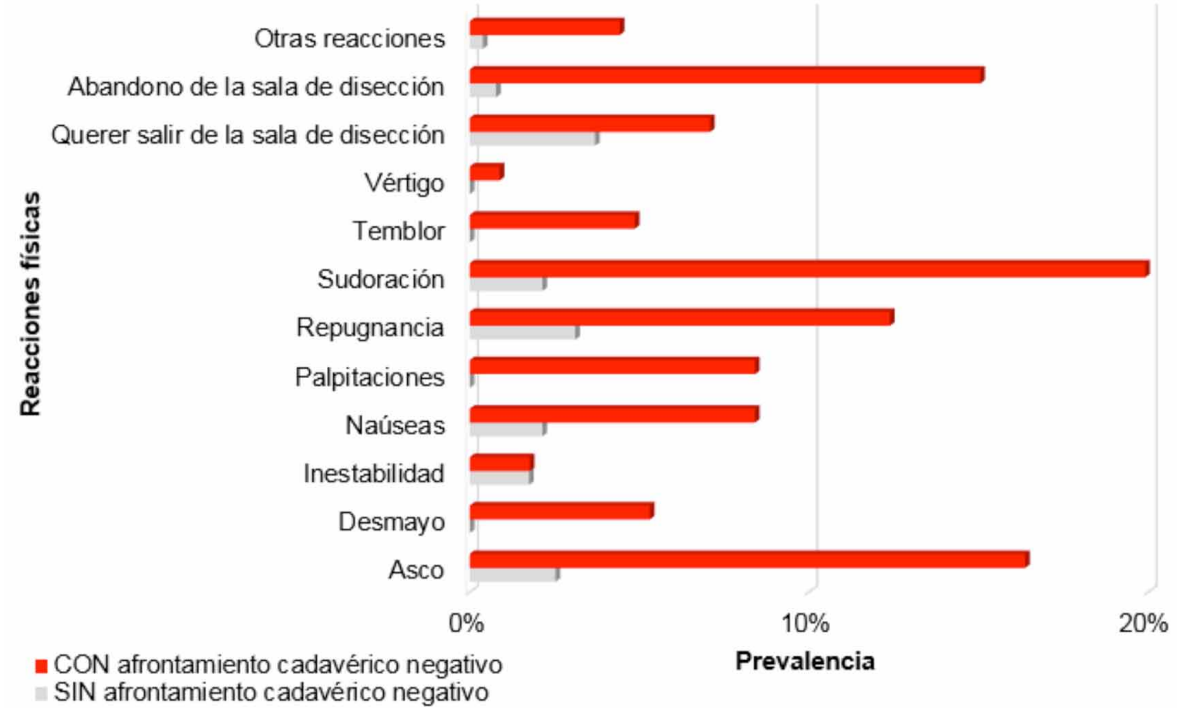

Fig. 5. Reacciones físicas con especificación de los grupos SIN y CON afrontamiento cadavérico negativo.

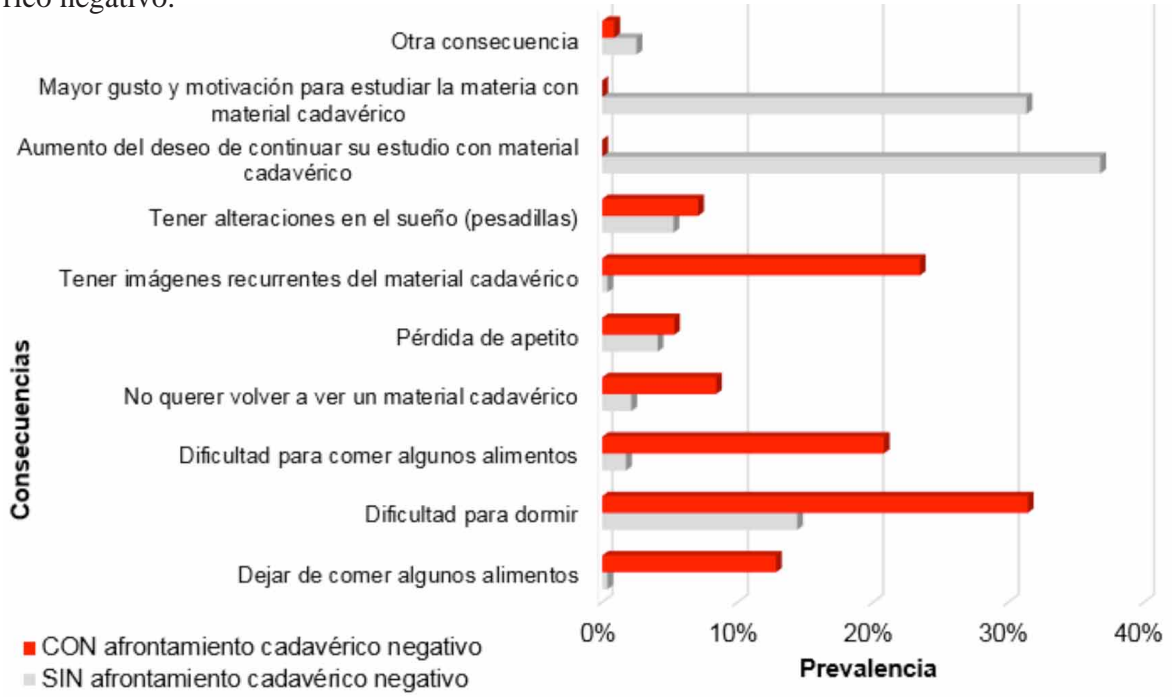

Fig. 6. Consecuencias frente a los TP con material cadavérico con especificación de los grupos SIN y CON afrontamiento cadavérico negativo. 
Ver la cara del material cadavérico

Ver intemamente un cuerpo humano

Ver el material cadavérico en piezas anatómicas o "desmembrado"

Ver el material cadavérico perteneciente a un ser

go
đo
므

$$
\text { humano }
$$

Ver alguna zona en particular del material cadavérico

Olor de la sala de trabajos prácticos

Olor del material cadavérico

Color del material cadavérico

Aspecto del material cadavérico
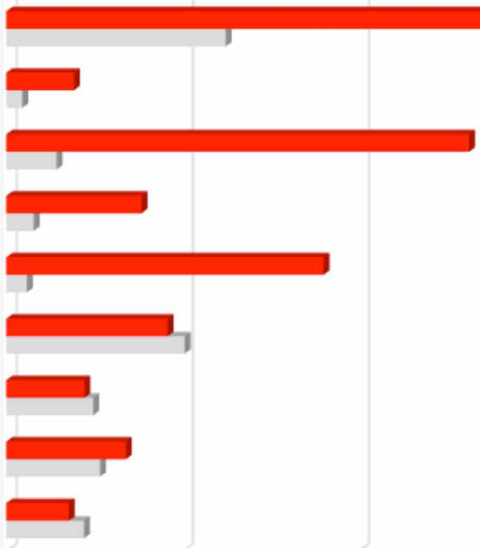

$0 \%$

- CON afrontamiento cadavérico negativo

SIN afrontamiento cadavérico negativo

$15 \%$

Prevalencia

Fig. 7. Impacto referido con especificación de los grupos SIN y CON afrontamiento cadavérico negativo.

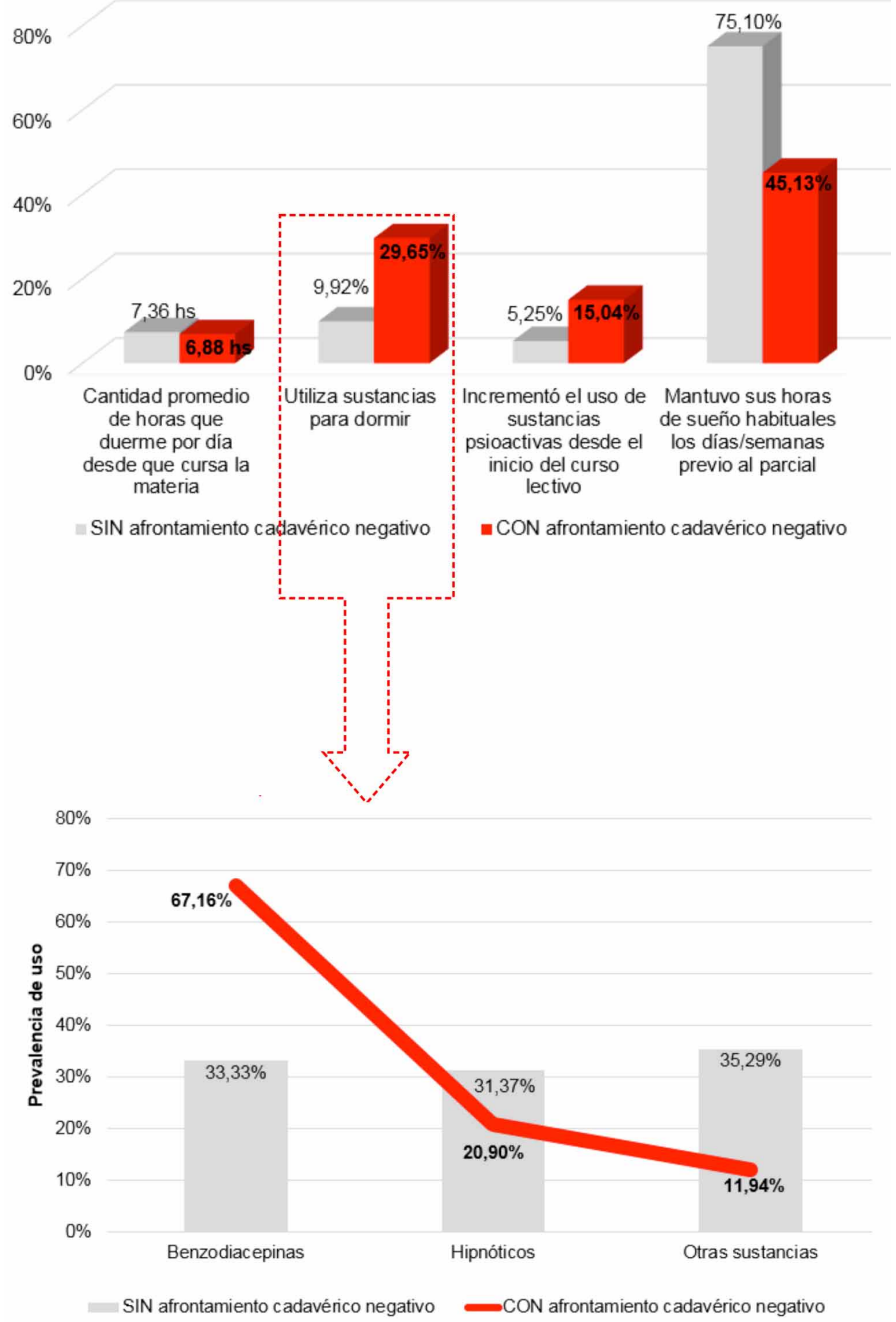

Fig. 8. Parámetros relacionados con el sueño y uso de sustancias para dormir, con especificación de los grupos SIN y CON afrontamiento cadavérico negativo. 


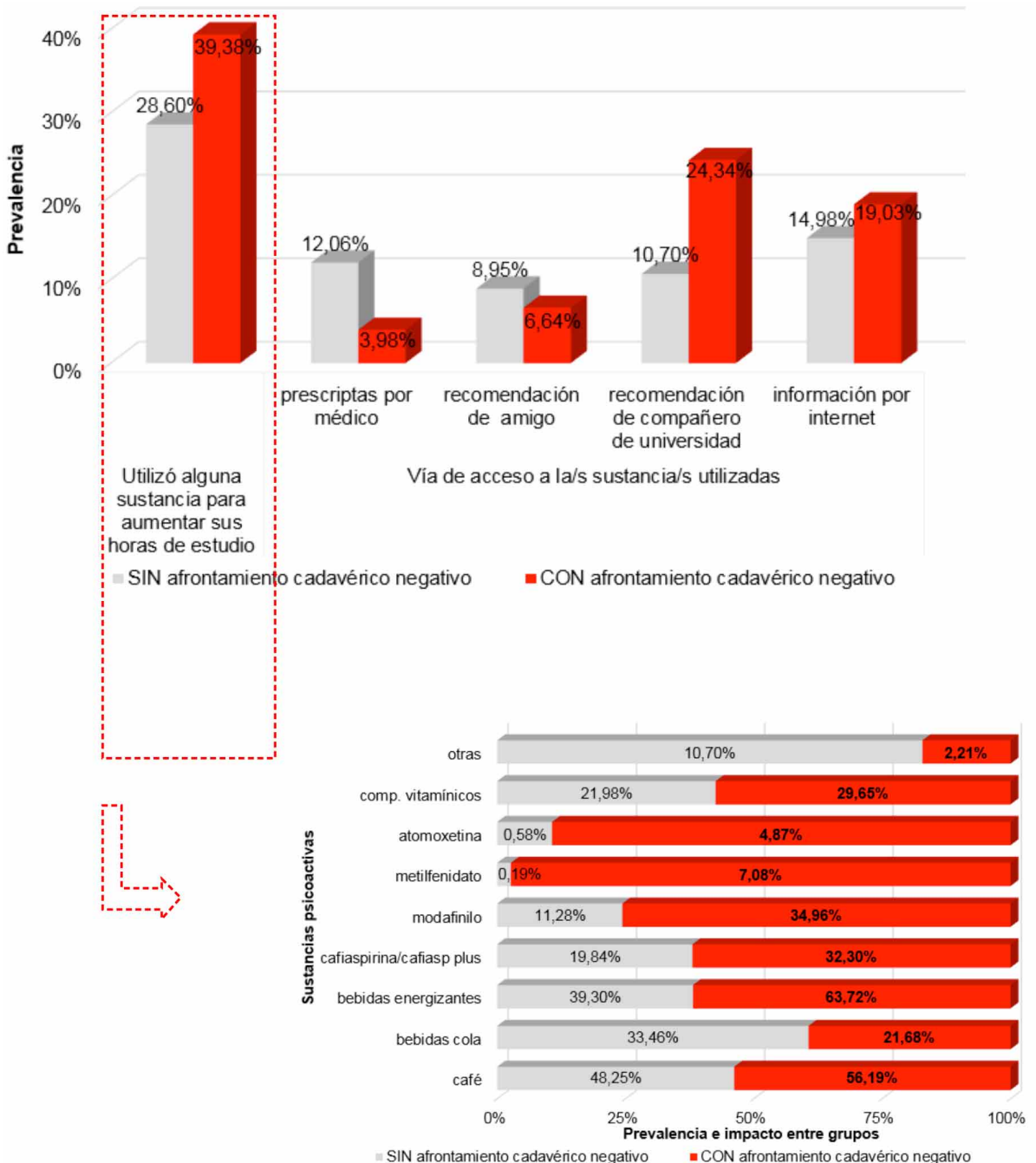

Fig. 9. Parámetros relacionados con el uso de sustancias psicoactivas para el estudio, con especificación de los grupos SIN y CON afrontamiento cadavérico negativo.

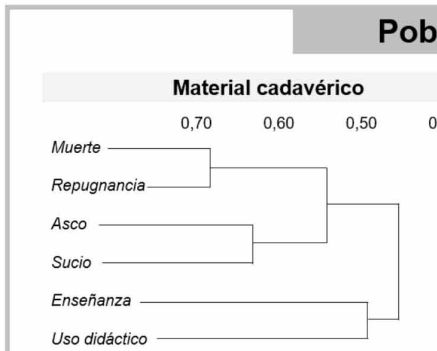

\section{Población SIN AfCN}

Uso didáctico

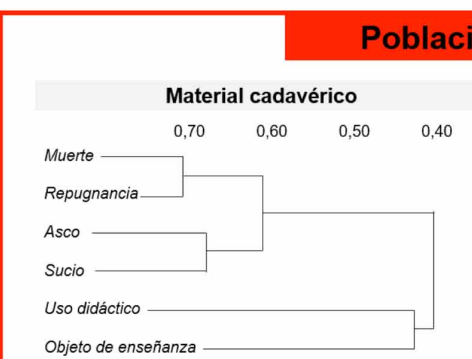

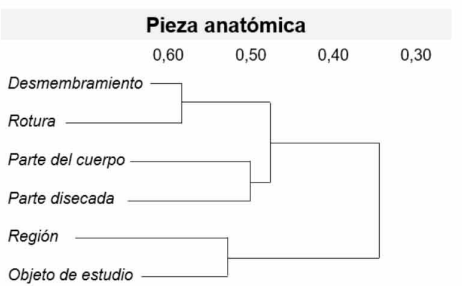

Objeto de estudio

- CON afrontamiento cadavérico negativo

Fig. 10. Dimensiones conceptuales por conglomerados jerárquicos de los términos analizados en población general y población con afrontamiento cadavérico negativo (AfCN). 

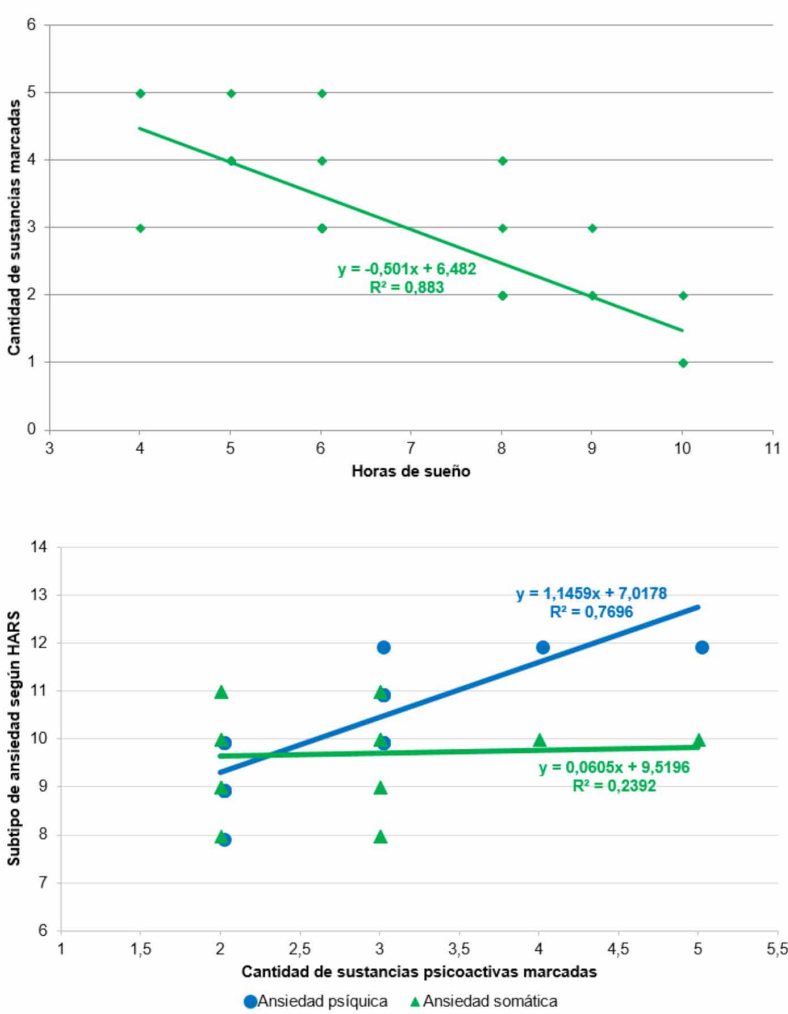

Fig. 11. Correlación entre cantidad de sustancias marcadas y las horas de sueño y el subtipo de ansiedad determinado por HARS.

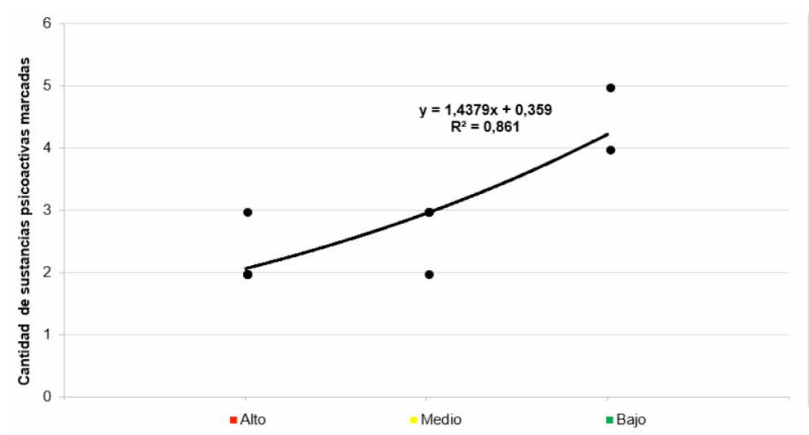

Fig. 12. Correlación entre la cantidad de sustancias utilizadas y el rango de ansiedad por HARS.

\section{DISCUSIÓN}

El proceso de enseñanza--aprendizaje basado en el uso de material cadavérico (MC) humano en la materia Anatomía se remonta a la enseñanza del Egipto antiguo durante el periodo helenístico. Actualmente, la enseñanza en los trabajos prácticos (TP) de la materia mantiene este tradicional recurso instruccional por sus ventajas didácticas, pero con pequeños cambios en la forma de presentación de cadáveres, formolización y disposición arquitectónica del aula. Se

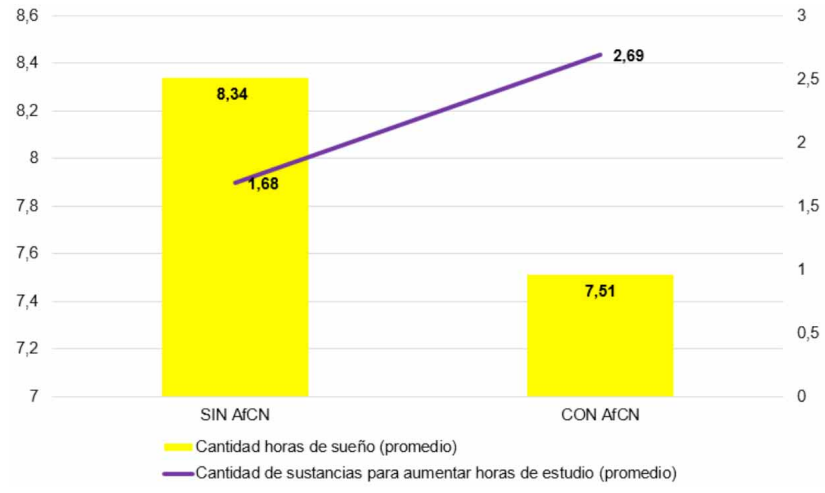

Fig. 13. Promedio de horas de sueño y de sustancias utilizadas para aumentar horas de estudio entre alumnos con y sin afrontamiento cadavérico negativo.

pasó de una enseñanza en anfiteatros en la cual un Maestro cirujano ejercía junto con sus colaboradores la enseñanza y mostración cadavérica a una dinámica pedagógica basada en mesas de TP con un auxiliar docente cada una.

La materia Anatomía es la primera de la Carrera de Medicina y conlleva muchas fantasías en los ingresantes así como en quienes no han estudiado medicina. Numerosas películas y series se centran en los devenires de los estudiantes durante su estudio, así como la complejidad de la misma. Es concebida como la antesala de la clínica, el cimiento fundacional de la enseñanza médica, así como también se la a relacionado con un "filtro" a ser atravesado para continuar con la carrera. La concepción de filtro se funda en su complejidad práctica y teórica, así como también una prueba a ser sorteada por quien desea ser médico. Si bien toda la carrera de Medicina es un devenir de experiencias estresantes (Snelling et al.; Arraéz-Aybar et al., 2004b; Pérez et al., 2007), el inicio de la carrera es un factor de distrés para el educando que debe ser afrontado por el mismo con sus mecanismos defensivos o bien con los que pueda ir desarrollando.

Para la mayoría de los alumnos que inician la cursada el estudio con MC es el primer acercamiento a un cuerpo humano muerto del cual, a través de su exploración, irán reconociendo estructuras, olores, colores y texturas. Implica un reto cognitivo el poder utilizar sincrónicamente varios sentidos (visual, táctil, olfatorio) en consonancia con funciones mentales superiores (visuoespacialidad, abstracción) para lograr un aprendizaje significativo mediante el MC utilizado. A esto se suma poder concatenar con contenidos mnésicos (muchos a corto plazo del estudio previo al TP) y la conceptualización de lo presenciado en las clases teóricas y presentaciones orales del auxiliar docente en simultáneo con el TP para introducir la temática a abordar en el TP. 
Este "primer encuentro" del estudiante con el MC puede generarle reacciones muy variadas: algunas relacionadas por la curiosidad y la novedad, otras con el aso y la repugnancia. Las reacciones en los alumnos son subjetivas y responden a una construcción tanto individual, asociada con la historia personal del sujeto, como colectiva o social relacionada con las concepciones e imaginarios sobre la muerte en una población. Tanto unas como otras se amalgaman y generan una respuesta emocional relacionada con el estrés. Las reacciones de los alumnos tienen injerencia no sólo en el aquí y ahora del TP, dado pueden generarles una mayor avidez por su estudio como también producir un alejamiento con el objeto de estudio o el abandono de la sala de disección, sino que son determinantes y hasta limitantes de la adherencia con la materia, con la carrera de Medicina, con el rendimiento académico, y producirá un impacto en el proceso de aprendizaje. Algunos investigadores como Kasper postularon que el afrontamiento cadavérico negativo se asociaría con la futura relación médico paciente. Basaron su hipótesis en el concepto descripto por Shaley y Nathan (Shalev \& Nathan, 1985) que asociaron la reacción con el MC como la "cristalización de la relación médico-paciente" y se resignificaría en las futuras situaciones conflictivas de la práctica asistencial. Por lo cual, el estrés y las reacciones emocionales que se despliegan durante el estudio con MC tendrían un impacto durante la cursada pero también en la vida profesional.

Estudios publicados a nivel internacional reportaron reacciones emocionales múltiples y heterogéneas con un núcleo común configurado por el estrés y los síntomas de ansiedad, que de prolongarse o ante sucesivas reexposiciones, puede asociarse con cuadros distímicos, conductuales y alteraciones cronobiológicas. Recién en el siglo XX, década del '60, aparecieron los primeros reportes y fue Fox (1979) quien describió el distrés reactivo a la disección de MC humano. Si bien muchos trabajos reportaron el estado emocional de los alumnos y su repercusión descripta por los síntomas referidos, así como signos evidenciados en el TP, no se ha profundizado en las reacciones de ansiedad reactiva, el impacto en la persona, sus factores de riesgo y el impacto pedagógico.

Las reacciones emocionales, negativas o positivas, son elaboradas por el alumno y en su mayoría son mitigadas o elaboradas. Pero existe un grupo de educandos que presenta un afrontamiento cadavérico negativo, es decir, una forma anómala de procesar las reacciones que le genera el estudio con el MC con una importante cantidad de síntomas que alteran su actividad académica, así como sus actividades de la vida diaria. Estudios de investigación reportaron una alta prevalencia de mecanis- mos que producen consecuencias negativas entre los estudiantes de Medicina (la evasión, idealización de situaciones, el aislamiento social y la autocrítica excesiva) y se reportó entre un 25 y $52 \%$ de depresión entre los estudiantes de Medicina. Otros estudios publicaron que entre los estudiantes de Medicina se halló una mayor tasa de consumo problemático de sustancias, principalmente alcohol, con el objetivo de disminuir los síntomas de ansiedad, estrés y síntomas depresivos. Nuestro equipo docente presentó en investigaciones previas (Mazzoglio y Nabar et al., 2011; Mazzoglio y Nabar et al., 2015b; Mazzoglio y Nabar et al., 2017a,b; Mazzoglio y Nabar et al., 2018) el significativo consumo de sustancias psicoactivas en esta población de primer año, destacándose el uso de ansiolíticos (benzodiacepinas) y activadores neuronales (bebidas energizantes y modafinilo), los cuales proveen de una respuesta sintomática rápida y efectiva en el corto plazo para disminuir los niveles de ansiedad, estrés, conciliar el sueño y/o aumentar las horas de vigilia destinadas al estudio, pero que son una conducta restitutiva que no aborda la/s causa/s subyacente/s y tienen efectos adversos que ponen en riesgo al alumno (dependencia, alteraciones de circuitos mnésicos). El uso de estos psicofármacos interfiere en los mecanismos de procesamiento y generación de la memoria dado alteran las redes atencionales, la producción de los engramas y la consolidación de redes asociativas, bases del aprendizaje significativo.

En el presente trabajo pudimos objetivar que los alumnos con afrontamiento cadavérico negativo presentaban una puntuación de ansiedad en rango moderado/ severo, con un cortejo sindromático caracterizado por síntomas autonómicos (palpitaciones, desmayos, temblores), síntomas cognitivos (falta de concentración), el estado de ánimo ansioso y alteraciones del sueño. En este grupo el uso de sustancias psicoactivas era mayor, principalmente benzodiacepinas, y la cantidad de sustancias utilizadas fue proporcional con la ansiedad psíquica.

Como docentes universitarios de una materia anual que se dicta en primer año de la carrera, consideramos que debemos tener en cuenta esta problemática por su prevalencia y factores de riesgo asociados. Asimismo, instaurar estrategias de enseñanza con foco en la innovación didáctica para disminuir estas reacciones y posibilitar el proceso de enseñanza-aprendizaje sin factores negativos. Actualmente nuestro equipo docente está llevando a cabo estrategias con diferentes recursos didácticos adaptados a la heterogeneidad de los alumnos y posibilidades horarias, espaciales y de recurso humano, con el fin de allanar el tránsito en los inicios de la carrera. 


\section{CONCLUSIONES}

Ratificamos la alta prevalencia de afrontamiento cadavérico negativo en alumnos que cursan Anatomía, el cual se asoció con un mayor nivel de ansiedad y uso de sustancias psicoactivas, principalmente ansiolíticos como respuesta restitutiva. Estos síntomas de ansiedad se asociaron con las dimensiones conceptuales registradas y tienen impacto en el proceso de enseñanza-aprendizaje.

MAZZOGLIO Y NABAR, M. J.; ALGIERI, R. D \& TORNESE, E. B. Anxiety and cadaveric coping in anatomy students. Int. J. Morphol., 37(3):928-937, 2019

SUMMARY: In preliminary studies we describe the clinical importance and pedagogical impact of the study with cadaverous material (MC) in Anatomy students and we found a significant using of psychoactive substances (SP), mainly repeating students and/or students with work activities. The form of cadaveric coping (AfC) is a factor of distress associated with symptoms (disgust, vomiting, sleep disturbances), with the use of SP and interferes with the educational process. The objective was to evaluate the anxiety in students with negative cadaveric coping. Observational and cross-sectional study by means of a survey of 740 students that included: Anxiety Scale against death -EAM-, Hamilton Anxiety Scale, questions about SP, and terms related to concepts ("cadaverous material", "anatomical piece") were investigated. In students with "negative cadaveric coping" (AfCN) (EAM <15, high / medium fear with CM and physical reactions) the Hamilton Anxiety Scale was applied. Statistical parameters, significance $\mathrm{p}<0.05$ and ethical-legal requirements were applied. We aimed at high and sustained prevalence of students with AfCN, who presented higher scores of anxiety and prevalence of SP use (anxiolytics with higher prevalence than excitatory substances); correlation was recorded between the amount of SP and the degree of negative reactions $(r=0.86)$. The psychic anxiety was greater and was associated with the degree of fear. We confirmed a high prevalence of AfCN that was associated with a higher level of anxiety and use of SP, mainly anxiolytics. The symptoms of anxiety were associated with the registered conceptual dimensions and have an impact on the teachinglearning process.

KEY WORDS: Anxiety Consumption of substances; Cadaveric coping; Anatomy; Academic stress.

\section{REFERENCIAS BIBLIOGRÁFICAS}

Arraéz-Aybar, L. A.; Casado-Morales, M. I. \& Castaño-Collado, G. Anxiety and dissection of the human cadaver: an unsolvable relationship? Anat. Rec. B New Anat., 279(1):16-23, 2004b.

Arraéz-Aybar, L. A.; Castaño-Collado, G. \& Casado-Morales, M. I. Dissection from the Spanish anatomist's perspective: aims, attitudes, and related aspects. Anat. Rec. B New Anat., 281(1):15-20, 2004a.

Babinski, M. A.; Sgrott, E. A.; Luz, H. P.; Brasil, F. B.; Chagas, M. A. \& AbiduFigueiredo, M. The relationship of the students with corpse in the pratical study of anatomy: the reaction and influence in the learning. Int. J. Morphol., 21(2):137-42, 2003.
Charlton, R.; Dovey, S. M.; Jones D. G. \& Blunt, A. Effects of cadaver dissection on the attitudes of medical students. Med. Educ., 28(4):290-5, 1994.

Fox, R. C. Essays in Medical Sociology: Journeys into the Field. New York, John Wiley, 1979.

Hamilton, M. The assessment of anxiety states by rating. Brit. J. Med. Psychol., 32(1):50-5, 1959.

Lobo, A.; Chamorro, L.; Luque, A.; Dal-Ré, R.; Badia, X. \& Baró E. Validación de las versiones en español de la Montgomery-Asberg Depression Rating Scale y la Hamilton Anxiety Rating Scale para la evaluación de la depresión y de la ansiedad. Med. Clin., 118(13):493-9, 2002.

Mazzoglio y Nabar, M. J.; Algieri, R. D.; Dogliotti, C. B.; Gazzotti, A. M.; Jiménez-Villarruel, H. N. \& Rey, L. M. Utilización de sustancias psicoactivas en alumnos de anatomía y su implicación en el aprendizaje. Educ. Med., 14(2):129-32, 2011.

Mazzoglio y Nabar, M. J.; Algieri, R. D.; Tornese, E. B.; Dogliotti, C. G.; Jiménez Villarruel, H. N.; Gazzotti, A. \& Ferrante, S. Psychoactive substances and mobile virtual spaces: impact on teaching and learning of anatomy. Int. J. Morphol., 33(4):1487-93, 2015 b.

Mazzoglio y Nabar, M. J.; Algieri, R. D.; Tornese, E. B.; Ferrante, M. S.; Broffman, C. \& Algieri, A. Consumo de Sustancias Psicoactivas en Cursantes de Anatomía con Afrontamiento Cadavérico Negativo. Corrientes, XXIII Congreso de la Asociación de Ciencias Morfológicas de Corrientes y XVIII Congreso Internacional, 2017a.

Mazzoglio y Nabar, M. J.; Rubio Domínguez, E.; Giordano, S.; Schraier, G.; Martínez Bernhardt, E. \& Algieri, R. D. Afrontamiento Cadavérico en Medicina: Asociación con Consumo de Sustancias Psicoactivas y Otras Conductas de Riesgo. Póster. Mar del Plata, XXXIII Congreso Argentino de Psiquiatría, 2018.

Mazzoglio y Nabar, M. J.; Rubio Domínguez, E.; Giordano, S.; Schraier, G.; Martínez Bernhardt, E. \& Algieri, R. D. Afrontamiento Cadavérico, Consumo de Sustancias Psicoactivas y Conductas de Riesgo en Alumnos de Primer Año. Buenos Aires, Facultad de Medicina, Universidad de Buenos Aires, $2017 \mathrm{~b}$.

Mazzoglio y Nabar, M. J.; Tornese, E. B.; Algieri, R. D.; Dogliotti, C. G.; Ferrante, M. S. \& Gómez, D. Afrontamiento cadavérico: respuestas subjetivas de alumnos de anatomía y su implicancia en el proceso de enseñanza y aprendizaje. Comunicación oral. Rosario, LII Congreso Argentino de Anatomía, 2015a. pp.88-9. Disponible en: http://www.revista-anatomia.com.ar/archivos-parciales/2015-4-supl-revista-argentina-de-anatomia-online-a.pdf

Mazzoglio y Nabar, M.; Algieri, R. D.; Tornese, E.; Dogliotti, C.; Ferrante, S.; Gazzotti, A.; Jiménez Villarruel, H.; Rey, L.; Alonso, H.; Gómez, D. \& Viñas, J. Afrontamiento cadavérico y uso de tecnología virtual: respuestas subjetivas de alumnos de Anatomía y su impacto en las estrategias de enseñanza. Rev. Argent. Anat. Online, 7(3):115-24, 2016.

Pérez, M. M.; Porta Riba, N.; Ortiz Sagristà, J. C.; Martínez, A. \& Götzens García, V. Anatomía Humana: estudio de las reacciones de los estudiantes de primero de medicina ante la sala de disección. Educ. Med., 10(2):10513, 2007.

Shalev, A. \& Nathan, H. Medical students' stress reactions to dissections. Isr. J. Psychiatry Relat. Sci., 22(1-2):121-33, 1985.

Snelling, J.; Sahai, A. \& Ellis, H. Attitudes of medical and dental students to dissection. Clin. Anat., 16(2):165-72, 2003.

Templer, D. I. The construction and validation of a Death Anxiety Scale. J. Gen. Psychol., 82(2d Half):165-77, 1970.

Dirección para correspondencia:

Martín Javier Mazzoglio y Nabar

Facultad de Medicina

Universidad Nacional de Buenos Aires

Paraguay, 2055, 4o piso, Sector Uriburu

CP 1121

Buenos Aires

ARGENTINA

Recibido : 22-10-2018

Aceptado: 20-02-2019

Email: mazzoglioynabar@hotmail.com 